\title{
Gérard-Levelt membranes
}

\author{
Eduardo Corel $^{*}$
}

\begin{abstract}
We present an unexpected application of tropical convexity to the determination of invariants for linear systems of differential equations. We show that the classical Gérard-Levelt lattice saturation procedure can be geometrically understood in terms of a projection on the tropical linear space attached to a subset of the local affine Bruhat-Tits building, that we call the Gérard-Levelt membrane. This provides a way to compute the true Poincaré rank, but also the Katz rank of a meromorphic connection without having to perform gauge transforms nor ramifications of the variable. We finally present an efficient algorithm to compute this tropical projection map, generalising Ardila's method for the case of the Bergman fan to the case of the tight-span of a valuated matroid.
\end{abstract}

\section{Contents}

1 Meromorphic connections 3

1.1 Gérard-Levelt's saturated lattices . . . . . . . . . . . . . 3

2 Tropical convexity and lattices 5

3 The Gérard-Levelt membranes 6

3.1 Tropical computation of the Katz rank . . . . . . . . . . . . . . 7

4 A projection algorithm on a tropical linear space 8

4.1 Valuated matroids . . . . . . . . . . . . . . 8

4.2 An algorithm for the projection on the tight-span . . . . . . . . 9

\section{Introduction}

Given a meromorphic linear differential system on the Riemann sphere,

$$
\frac{d X}{d z}=A(z) X \text { with } A(z) \in \mathrm{M}_{n}(\mathbb{C}(z))
$$

it is important to determine whether a singularity of $A$ is a regular singular point for the system (1). Unlike with scalar linear differential equations, for which there is a purely algebraic condition on the orders of the poles of the coefficients due to L. Fuchs $[\mathrm{Fu}$, a system (11) can display arbitrarily high pole orders at a regular singularity.

Consider the differential system, expanded in the neighbourhood of the singular point (assumed for simplicity to be $z=0$ ) as follows, where we put $\theta=z \frac{d}{d z}$,

$$
\theta X=\frac{1}{z^{p}} \sum_{i \geqslant 0} A_{i} z^{i} X \text { with } p \geqslant 0 \text { and } A_{0} \neq 0 \text { if } p>0 .
$$

\footnotetext{
*E-mail : ecorel@gwdg.de
} 
The integer $p$ is traditionally known as the Poincaré rank $\mathfrak{p}(A)$ of the system. Finding the type of singularity involves knowing the minimum value $m(A)$, sometimes known as the true Poincaré rank, of this rank under gauge transformations

$$
A_{[P]}=P^{-1} A P-P^{-1} \theta P \text { with } P=\sum_{k \geqslant k_{0}} P_{k} z^{k} \in \mathrm{GL}_{n}(K) \text { where } K=\mathbb{C}((z)) .
$$

Several lines of research have been opened to tackle this problem. The most classical tries to iteratively construct a suitable gauge transformation $P$, usually coefficient by coefficient in the series expansion. Featured methods rely on the linear algebra over $\mathbb{C}$ involved by equation (3), like Moser and continuators ([Mo, $\mathrm{H}-\mathrm{W}]$ ), whose methods are widely used nowadays in computer algebra, or other researchers such as Ju, Ba-J-L, while [B-V] use Lie group theoretic tools.

The nature of a singularity of $A$ can also considered from point of view of meromorphic connections [De, and especially, as a question of stability of certain lattices under the differential operator induced by the connection $[\mathrm{M}, \mathrm{Ka}$. We focus here specifically on the approach of saturating lattices used by Gérard and Levelt [G-L]: the true Poincaré rank $m(\nabla)$ is the minimum integer $k$ such that the sequence of $k$-saturated lattices (recalled in section 1.1) eventually stabilises.

Recent work has shown close relations between the geometric framework of the Bruhat-Tits building of SL $(K)$, for some discrete valued field such as $K=\mathbb{C}((z))$, and tropical convexity [K-T, J-S-Y, W]. We show here that Gérard and Levelt's approach can be formulated and efficiently computed in this framework, as a projection on the tropical linear space $L_{p}$ attached to the valuated matroid $p$ corresponding to a given membrane in the Bruhat-Tits building.

Results of Keel and Tevelev [K-T] show that, when lattices are in a same membrane of the Bruhat-Tits building, they are homothetic if and only if they are projected on the same point of a tropical linear space by an explicit nearest point projection map ([J-S-Y], see also C-G-Q, G-K] ). We need to be able to tell whether lattices are actually equal. We show that the projection map respects the valuation, and projects only equal lattices on a same point (theorem 2 ).

We then construct the Gérard-Levelt membrane that contains all the $k$-saturated lattices (proposition 1), and give a tropical version of Gérard-Levelt's lattice stabilisation criterion (corollary 1). Third, we show that the same formula amazingly allows for the computation of a more subtle invariant, namely the Katz rank of the connection (theorem 3), without having to either to compute a single gauge transformation nor perform the usually required ramification of the variable.

The projection map given in [J-S-Y] has unfortunately a high complexity. We give in section 4 an efficient algorithm to compute this projection map onto the linear tropical space attached to a valuated matroid. We generalise the algorithmic approach to tropical projection developed by Ardila $\mathrm{Ar}$ for ordinary matroids, to the case of valuated matroids defined by Dress and collaborators [D-W]. The algorithm presented here, which computes the nearest $\ell_{\infty}$-projection on the tight-span of a valuated matroid, has a wider applicability than the differential computations explained in the previous parts, especially in phylogenetics [D-T].

\section{Acknowledgements}

I have benefited from very interesting and stimulating discussions with Federico Ardila, Josephine Yu, Michael Joswig, Annette Werner, Felipe Rincón and Stéphane 
Gaubert, held at the Tropical Geometry Workshop at the CIEM in Castro Urdiales (Spain) in December 2011.

\section{Meromorphic connections}

A meromorphic connection is a map $\nabla: V \simeq K^{n} \longrightarrow \Omega(V)=V \otimes_{K} \Omega_{\mathbb{C}}^{1}(K)$ which is $\mathbb{C}$-linear and satisfies the Leibniz rule

$$
\nabla(f v)=v \otimes d f+f \nabla v \text { for } f \in K \text { and } v \in V .
$$

The matrix $\operatorname{Mat}(\nabla,(e))$ is given by $\nabla e_{j}=-\sum_{i=1}^{n} e_{i} \otimes \Omega_{i j}$ for a basis (e). A basis change $P \in \mathrm{GL}_{n}(K)$ gauge-transforms the matrix of $\nabla$ by

$$
\Omega_{[P]}=P^{-1} \Omega P-P^{-1} d P .
$$

Contracting with $z^{k+1} \frac{d}{d z}$ yields a differential operator $\nabla_{k}$, and system (1) is the expression of $\nabla_{-1}(v)=0$ in the basis $(e)$.

A lattice $\Lambda$ in $V$ is a free sub-O-module of rank $n$, that is a module of the form

$$
\Lambda=\bigoplus_{i=1}^{n} \mathcal{O} e_{i} \text { for some basis }(e) \text { of } V .
$$

The Poincaré rank of $\nabla$ on the lattice $\Lambda$ is defined as the integer

$$
\mathfrak{p}_{\Lambda}(\nabla)=-v_{\Lambda}\left(\Lambda+\nabla_{0}(\Lambda)\right)=\min \left\{k \in \mathbb{N} \mid \nabla_{k}(\Lambda) \subset \Lambda\right\}=\max _{i, j}\left(-v\left(\Omega_{i j}\right)-1,0\right) .
$$

The true Poincaré rank $m(\nabla)=\min _{\Lambda} \mathfrak{p}_{\Lambda}(\nabla)$ characterises the nature of the singularity of (1D), in the sense that $z=0$ is regular if and only if $m(\nabla)=0$.

\subsection{Gérard-Levelt's saturated lattices}

For any vector $e \in V$ and any derivation $\tau \in \operatorname{Der}(K / \mathbb{C})$, define for $\ell \in \mathbb{N}$ the family

$$
Z_{\tau}^{\ell}(e)=\left(e, \nabla_{\tau} e, \ldots, \nabla_{\tau}^{\ell} e\right) .
$$

The module $\mathcal{O}_{\tau}^{\ell}(e)$ induced over $\mathcal{O}$ by $Z_{\tau}^{\ell}(e)$ only depends on the valuation $v(\tau)$ of the derivation $\tau$. We can therefore restrict ourselves to the particular derivations $\tau_{k}=z^{k+1} \frac{d}{d z}$ for $k \in \mathbb{N}$. In this case, we put $\nabla_{\tau_{k}}=\nabla_{k}$, and note $Z_{k}^{\ell}(e)$ and $\mathcal{O}_{k}^{\ell}(e)$ the corresponding objects. For $k \geqslant 1$, Gérard and Levelt define the lattices

$$
F_{k}^{\ell}(\Lambda)=\Lambda+\nabla_{k} \Lambda+\cdots+\nabla_{k}^{\ell} \Lambda
$$

Note that

$$
F_{k}^{\ell}(\Lambda)=\sum_{i=1}^{n} Z_{k}^{\ell}\left(e_{i}\right) \text { for any basis }\left(e_{1}, \ldots, e_{n}\right) \text { of } \Lambda .
$$

Theorem 1 (Gérard, Levelt). The true Poincaré rank $m(\nabla)$ of $\nabla$ is

$$
m(\nabla)=\min \left\{k \in \mathbb{N} \mid F_{k}^{n}(\Lambda)=F_{k}^{n-1}(\Lambda)\right\} \text { for any lattice } \Lambda \subset V .
$$


This means that $k \geqslant m(\nabla)$ if and only if $\nabla_{k}\left(F_{k}^{n-1}(\Lambda)\right) \subset F_{k}^{n-1}(\Lambda)$ for some (equivalently, any) lattice $\Lambda$ in $V$, that is, that the Poincaré rank on $F_{k}^{n-1}(\Lambda)$ is at most $k$. Stated otherwise, finding the true Poincaré rank is finding the largest lattice whose Poincaré rank is bounded by its index in the following sequence

$$
F_{0}^{n-1}(\Lambda) \supset \cdots \supset F_{p-1}^{n-1}(\Lambda) \supset \Lambda
$$

Let us extend this notation to multi-indices. Let $\ell \geqslant 0$, and let $\alpha=\left(\alpha_{1}, \ldots, \alpha_{\ell}\right) \in$ $\mathbb{Z}^{\ell}$ be an integer multi-index of length $|\alpha|=\ell$ and weight $w(\alpha)=\alpha_{1}+\cdots+\alpha_{\ell}$. Let us define also the partial multi-indices $\alpha_{\mid j}=\left(\alpha_{1}, \ldots, \alpha_{j}\right)$ and

$$
\nabla^{\alpha}=\nabla_{\alpha_{\ell}} \circ \cdots \circ \nabla_{\alpha_{1}}
$$

Let by convention $\alpha_{\mid 0}=\epsilon$ and $\nabla^{\epsilon}=\mathrm{id}_{V}$ for the empty sequence $\epsilon$. Let finally $\mathcal{O}^{\alpha}(e)$ be the $\mathcal{O}$-module spanned by the sequence

$$
Z^{\alpha}(e)=\left(\nabla^{\alpha \mid j} e\right)_{0 \leqslant j \leqslant|\alpha|} .
$$

Lemma 1. For any $\alpha=\left(\alpha_{1}, \ldots, \alpha_{\ell}\right) \in \mathbb{Z}^{\ell}$, one has

$$
\nabla^{\alpha}=z^{w(\alpha)} P_{\alpha}\left(\nabla_{0}\right) \text { where } P_{\alpha}(X)=X\left(X+\alpha_{1}\right) \cdots\left(X+w\left(\alpha_{\mid \ell-1}\right)\right) \in \mathbb{Z}[X] .
$$

Proof. The proof goes by induction on the length of the multi-index $\alpha$. Let $D=\nabla_{0}$. The claim obviously holds for a multi-index of length 0 , with $P_{\epsilon}=1$, so assume that there exists $P_{\alpha} \in \mathbb{Z}[X]$ such that $\nabla^{\alpha}=z^{w(\alpha)} P_{\alpha}(D)$ for $|\alpha| \leqslant \ell$. Let $\beta \in \mathbb{Z}^{\ell+1}$. Then by definition, we have

$$
\begin{aligned}
\nabla^{\beta} & =z^{\beta_{\ell+1}} D \circ \nabla^{\beta_{\mid \ell}} \\
& =z^{\beta_{\ell+1}} D \circ\left(z^{w\left(\beta_{\mid \ell)}\right.} P_{\beta_{\mid \ell}}(D)\right) \\
& =z^{\beta_{\ell+1}}\left(w\left(\beta_{\mid \ell}\right) z^{w\left(\beta_{\mid \ell)}\right.} P_{\beta_{\mid \ell}}(D)+z^{w\left(\beta_{\mid \ell)}\right.} D \circ P_{\beta_{\mid \ell}}(D)\right) \\
& =z^{w(\beta)}\left(w\left(\beta_{\mid \ell}\right) P_{\beta_{\mid \ell}}(D)+P_{\beta_{\mid \ell}}(D) \circ D\right) \\
& =z^{w(\beta)}\left(P_{\beta_{\mid \ell}}(D) \circ\left(D+w\left(\beta_{\mid \ell}\right)\right)\right)
\end{aligned}
$$

Indeed, $P_{\beta_{\mid \ell}}(D)$ commutes with $D$ since it has by assumption constant coefficients. The result follows, since we have then $P_{\beta}(X)=P_{\beta_{\mid \ell}}(X)\left(X+w\left(\beta_{\mid \ell}\right)\right)$.

Lemma 2. Let $\Lambda$ be a lattice in $V$. For any $\ell \in \mathbb{N}$ and $\alpha \in \mathbb{N}^{\ell}$, the $\mathcal{O}$-module $\mathcal{O}^{\alpha}(e)$ is spanned over $\mathcal{O}$ by the family

$$
\left(e, z^{\alpha_{1}} \nabla_{0} e, \ldots, z^{w\left(\alpha_{\mid \ell-1}\right)} \nabla_{0}^{\ell-1} e\right) .
$$

Proof. According to lemma 1, the family $Z_{\alpha}^{\ell-1}(e)$ is related to $Z_{0}^{\ell-1}(e)$ by the matrix $P=A z^{W_{\alpha}}$ where

$$
W_{\alpha}=\operatorname{diag}\left(0, \alpha_{1}, \ldots, w\left(\alpha_{\mid \ell-1}\right)\right)
$$

and $A$ is an upper triangular integer matrix with diagonal entries equal to 1 , therefore $A \in \mathrm{SL}_{\ell}(\mathbb{Z}) \subset \mathrm{GL}_{\ell}(\mathcal{O})$. The families $z^{W_{\alpha}} Z_{0}^{\ell-1}(e)$ and $Z_{\alpha}^{\ell-1}(e)$ are related by the matrix

$$
\tilde{P}=z^{-W_{\alpha}} P=z^{-W_{\alpha}} A z^{W_{\alpha}} \text { whose entries are } A_{i j} z^{w\left(\alpha_{\mid j}\right)-w\left(\alpha_{\mid i}\right)} .
$$

Since $A$ is upper triangular, and the partial sums $\alpha_{i}+\cdots+\alpha_{j}$ are non-negative, the matrix $\tilde{P}$ is in $\mathrm{GL}_{n}(\mathcal{O})$, and therefore both families span the same $\mathcal{O}$-module. 


\section{Tropical convexity and lattices}

Let $M=\left\{d_{1}, \ldots, d_{m}\right\}$ be lines in $V$ such that $d_{1}+\cdots+d_{m}=V$, and consider the subset of $\boldsymbol{\Lambda}$ defined by

$$
[M]=\left\{\ell_{1}+\cdots+\ell_{m} \mid \ell_{i} \text { is a lattice in } d_{i}\right\} .
$$

Following Keel and Tevelev, who call in $[\mathrm{K}-\mathrm{T}]$ the set induced by $[M]$ modulo homothety a membrane, we call this the affine membrane spanned by $M$.

For a choice $\mathcal{A}=\left(v_{1}, \ldots, v_{m}\right)$ of non-zero vectors in the lines $d_{i}$, any lattice in the membrane defined by $M=\left\{d_{1}, \ldots, d_{m}\right\}$ can be represented (non uniquely) by an integer valued point as follows: a lattice point $u \in \mathbb{Z}^{m}$ corresponds to the lattice

$$
\Lambda_{u}=\sum_{i=1}^{m} \mathcal{O} z^{-u_{i}} v_{i}
$$

Membranes spanned by $m$ lines in the Bruhat-Tits building have a faithful representation as tropical linear spaces in $m$-dimensional space.

Let $\left(\mathbb{R}_{\infty}=\mathbb{R} \cup\{\infty\}, \oplus, \odot\right)$ be the tropical semialgebra, where the operations are

$$
x \oplus y=\min (x, y) \text { and } x \odot y=x+y \text { for } x, y \in \mathbb{R}_{\infty} .
$$

An affine membrane $M$ and a basis $(e)$ of $V$ determine a valuated matroid

$$
\begin{aligned}
p:[m]^{n} & \longrightarrow \mathbb{R}_{\infty} \\
\omega & \longmapsto v\left(\operatorname{det}_{(e)} M_{\omega}\right)
\end{aligned}
$$

where $M_{\omega}=\left(v_{\omega_{1}}, \ldots, v_{\omega_{n}}\right)$ is the subfamily of vectors of $M$ indexed by $\omega$. To a valuated matroid $p$ of rank $n$ over $[m]$ there is a tropical linear space $L_{p} \subset \mathbb{R}_{\infty}^{m}$ attached as follows

$$
L_{p}=\left\{x \in \mathbb{R}_{\infty}^{m} \mid \forall \tau \in\left(\begin{array}{c}
{[m]} \\
n+1
\end{array}\right), \min _{1 \leqslant i \leqslant n+1} p\left(\tau \backslash\left\{\tau_{i}\right\}\right)+x_{\tau_{i}} \text { is attained twice }\right\} .
$$

Depending on the authors, $L_{p}$ is said to be a tropical convex cone ([C-G-Q]) or a convex polytope $([\mathrm{J}-\mathrm{S}-\mathrm{Y}])$ in $\mathbb{R}_{\infty}^{m}$. Both definitions mean that

$$
\lambda \odot u \oplus \mu \odot v \in L_{p} \text { for any } \lambda, \mu \in \mathbb{R}_{\infty} \text { and } u, v \in L_{p} .
$$

According to $\mathrm{C}-\mathrm{G}-\mathrm{Q}, \mathrm{G}-\mathrm{K}, \mathrm{J}-\mathrm{S}-\mathrm{Y}$, for $x \in \mathbb{R}_{\infty}^{m}$, the formula

$$
\pi_{L_{p}}(x)=\min \left\{w \in L_{p} \mid w \geqslant x\right\} \text { where the minimum is taken coordinate-wise, }
$$

defines the nearest point projection map $\pi_{L_{p}}: \mathbb{R}_{\infty}^{m} \longrightarrow L_{p}$. There are at least two other known ways to characterise or compute $\pi_{L_{p}}(x)$.

Blue Rule. Adapting $\left[\mathrm{Ar}\right.$, the authors of $[\mathrm{J}-\mathrm{S}-\mathrm{Y}]$ show that $\pi_{L_{p}}(x)=\left(w_{1}, \ldots, w_{m}\right)$ with

$$
w_{i}=\min _{\sigma \in\left(\begin{array}{l}
{[m]} \\
n-1
\end{array}\right)} \max _{j \neq \sigma}\left(p(\sigma \cup\{i\})-p(\sigma \cup\{j\})+x_{j}\right) .
$$


Red Rule. Similarly, starting with $v=(0, \ldots, 0) \in \mathbb{R}^{m}$, for every $\tau \in\left(\begin{array}{c}{[m]} \\ n+1\end{array}\right)$ such that $\alpha=\min _{1 \leqslant i \leqslant n+1} p\left(\tau \backslash\left\{\tau_{i}\right\}\right)+x_{\tau_{i}}$ is only attained once, say at $\tau_{i}$, compute $\gamma=\beta-\alpha$ where $\beta$ is the second smallest number in that collection, and put $v_{\tau_{i}}:=\max \left(v_{\tau_{i}}, \gamma\right)$. Then $\pi_{L_{p}}(x)=x+v$.

Theorem 2 (Keel-Tevelev). The nearest point projection map $\pi_{L_{p}}: \mathbb{R}_{\infty}^{m} \longrightarrow L_{p}$ induces a bijection $\Psi_{M}$ between $[M]$ and the lattice points in $L_{p}$

$$
\Psi_{M}\left(\Lambda_{u}\right)=\pi_{L_{p}}\left(u_{1}, \ldots, u_{m}\right) .
$$

In particular, $M_{u}=\left(z^{-u_{1}} v_{1}, \ldots, z^{-u_{m}} v_{m}\right)$ and $M_{u^{\prime}}=\left(z^{-u_{1}^{\prime}} v_{1}, \ldots, z^{-u_{m}^{\prime}} v_{m}\right)$ span the same lattice $\Lambda$ if and only if $\pi_{L_{p}}(u)=\pi_{L_{p}}\left(u^{\prime}\right)$.

Proof. Let $\Lambda=\sum_{i=1}^{m} \mathcal{O} z^{-u_{i}} v_{i}$, and let $w=\pi_{L_{p}}(u)$. According to $[\underline{\mathrm{K}}-\mathrm{T}$, th. 4.17 (see also [J-S-Y] th. 18), there exists $\alpha \in \mathbb{R}$ such that $w_{i}=v_{\Lambda}\left(v_{i}\right)+\alpha$ for all $1 \leqslant i \leqslant m$. By definition, $v_{\Lambda}(x)=\max \left\{k \in \mathbb{Z} \mid x \in z^{k} \Lambda\right\}$. Accordingly, we have $z^{-w_{i}} v_{i} \in z^{-\alpha} \Lambda$, and thus $\alpha \geqslant 0$. By formula (9), we get $\alpha=0$ and thus

$$
\pi_{L_{p}}(u)=\left(v_{\Lambda}\left(v_{1}\right), \ldots, v_{\Lambda}\left(v_{m}\right)\right) .
$$

By construction, if $u^{\prime} \geqslant u$, then $z^{-u_{i}} v_{i}=z^{\left(u_{i}^{\prime}-u_{i}\right)} z^{-u_{i}^{\prime}} v_{i} \in \mathcal{O} z^{-u_{i}^{\prime}} v_{i}$, for $1 \leqslant i \leqslant m$, hence $\Lambda \subset \Lambda_{u^{\prime}}$. Since in particular $w \geqslant u$ holds, we get $\Lambda \subset \Lambda_{w}$. Conversely, we have $\Lambda_{w}=\sum_{i=1}^{m} \mathcal{O} z^{-w_{i}} v_{i} \subset \Lambda$. Therefore, if $\pi_{L_{p}}(u)=\pi_{L_{p}}\left(u^{\prime}\right)$ then $\Lambda_{u}=\Lambda_{u^{\prime}}$. The converse follows directly from (10).

\section{The Gérard-Levelt membranes}

Proposition 1. Fix a basis (e) of $\Lambda$, and $\ell \geqslant 0$. Let $\left[M_{\ell}\right]$ be the membrane spanned by the vectors $\left(\nabla_{0}^{j} e_{i}\right)_{1 \leqslant i \leqslant n, 0 \leqslant j \leqslant \ell}$. Then $F_{k}^{\ell^{\prime}}(\Lambda) \in\left[M_{\ell}\right]$ for all $k \geqslant 0$ and $\ell^{\prime} \leqslant \ell$.

Proof. For the considered basis $(e)$, the lattice $L=F_{k}^{\ell^{\prime}}(\Lambda)$ satisfies

$$
L=\mathcal{O}^{\alpha}\left(e_{1}\right)+\cdots+\mathcal{O}^{\alpha}\left(e_{n}\right) \text { with } \alpha=\left(0, k, \ldots, k \ell^{\prime}\right) .
$$

Reordering terms as $\left(e_{1}, \ldots, e_{n}, \nabla_{0} e_{1}, \ldots \nabla_{0} e_{n}, \ldots, \nabla_{0}^{\ell^{\prime}} e_{n}\right)$, formula (7) and lemma 2 imply that $L$ can be represented in the membrane $\left[M_{\ell^{\prime}}\right]$ by the lattice point

$$
(\underbrace{0, \ldots, 0}_{n \text { times }}, \underbrace{-k, \ldots,-k}_{n \text { times }}, \ldots, \underbrace{-k \ell^{\prime}, \ldots,-k \ell^{\prime}}_{n \text { times }}) .
$$

Since by definition, $z^{-v_{\Lambda}(v)} v \in \Lambda$ holds for any $v \in V$, the module $L$ can also be represented as an element of the membrane $\left[M_{\ell}\right]$ by

$$
(\underbrace{0, \ldots, 0}_{n \text { times }}, \underbrace{-k, \ldots,-k}_{n \text { times }}, \ldots, \underbrace{-k \ell^{\prime}, \ldots,-k \ell^{\prime}}_{n \text { times }}, v_{\Lambda}\left(\nabla_{0}^{\ell^{\prime}+1} e_{1}\right), \ldots, v_{\Lambda}\left(\nabla_{0}^{\ell} e_{n}\right)) .
$$

The lattices $F_{k}^{\ell}(\Lambda)$ for $0 \leqslant \ell \leqslant n$ can therefore all be seen as elements of the same membrane $\left[M_{n}\right]$.

Definition 1. $\mathcal{M}_{\Lambda}=\left[M_{n}\right]$ is called the Gérard-Levelt membrane attached to $\Lambda$. For any basis $(e)$, the lattice $F_{k}^{\ell}(\Lambda)$ is represented by the lattice point

$$
u_{k}^{\ell}=(\underbrace{0, \ldots, 0}_{n \text { times }}, \underbrace{-k, \ldots,-k}_{n \text { times }}, \ldots, \underbrace{-k \ell, \ldots,-k \ell}_{n \text { times }}, v_{\Lambda}\left(\nabla_{0}^{\ell+1} e_{1}\right), \ldots, v_{\Lambda}\left(\nabla_{0}^{n} e_{n}\right)) .
$$


If $\operatorname{Mat}\left(\nabla_{0},(e)\right)=A$ for an basis $(e)$ of $\Lambda$, then $\mathcal{M}_{\Lambda}$ is described in $(e)$ by the $n \times n(n+1)$ matrix

$$
\mathbf{M}=\left(\begin{array}{llll}
I_{n} & A & \cdots & A_{n}
\end{array}\right) \text { where } A_{k+1}=\left(z \frac{d}{d z}+A\right) A_{k} \text { and } A_{0}=I_{n} .
$$

The tropical projection $\pi_{\Lambda}$ onto the tropical linear space $L_{\Lambda}$ attached to the Gérard-Levelt membrane $\mathcal{M}_{\Lambda}$ maps a point $u$ to a unique representative. Checking if $k \geqslant m(\nabla)$ requires to know if the lattice points $u_{k}^{n-1}$ and $u_{k}^{n}$ represent the same lattice, that is

$$
\pi_{\Lambda}\left(u_{k}^{n}\right)=\pi_{\Lambda}\left(u_{k}^{n-1}\right) .
$$

Corollary 1. For any $\Lambda$, we have $m(\nabla)=\min \left\{k \in \mathbb{N} \mid \pi_{\Lambda}\left(u_{k}^{n}\right)=\pi_{\Lambda}\left(u_{k}^{n-1}\right)\right\}$.

\subsection{Tropical computation of the Katz rank}

The tropical setting is compatible with the ramification of the variable. This implies the following result.

Theorem 3. Let $\pi_{\Lambda}: \mathcal{M}_{\Lambda} \longrightarrow L_{\Lambda}$ be the tropical nearest point projection map of the Gérard-Levelt membrane $\mathcal{M}_{\Lambda}$ of any lattice $\Lambda$ onto its attached tropical linear space $L$. Then the Katz rank $\kappa(\nabla)$ of the connection $\nabla$ satisfies

$$
\kappa(\nabla)=\min \left\{k \in \mathbb{R}^{+} \mid \pi_{\Lambda}\left(u_{k}^{n}\right)=\pi_{\Lambda}\left(u_{k}^{n-1}\right)\right\} \text { for any lattice } \Lambda .
$$

Proof. The Katz rank is the minimum Poincaré rank of the connection $\nabla_{H}$ induced on the pure algebraic extension $H=K[T] /\left(T^{N}-z\right)$ of $K$ with $N=\operatorname{lcm}(1,2, \ldots, n)$ (see e.g. [Cor] $)$. If we put $\zeta$ for the class of $T$, then $\operatorname{Mat}\left(\left(\nabla_{H}\right)_{\zeta \frac{d}{d \zeta}},(e \otimes 1)\right)=$ $N$ Mat $\left(\nabla_{z \frac{d}{d z}},(e)\right)$. Thus if $X(z)$ satisfies $z \frac{d}{d z} X(z)=A(z) X(z)$ the system satisfied by $Y(\zeta)=X\left(\zeta^{N}\right)$ is

$$
\zeta \frac{d}{d \zeta} Y(\zeta)=N A\left(\zeta^{N}\right) Y(\zeta)
$$

Put $\tilde{A}(\zeta)=N A\left(\zeta_{\tilde{A}}^{N}\right)$. The sequence $\left(\tilde{A}_{k}\right)_{k \in \mathbb{N}}$ defined by relation (11) of iterated $\zeta \frac{d}{d \zeta}$-derivatives of $\tilde{A}$ satisfies

$$
\tilde{A}_{k}(\zeta)=N^{k} A_{k}\left(\zeta^{N}\right)
$$

Let $q$ be the valuated matroid defined by $q(\omega)=w\left(\operatorname{det} \tilde{M}(\zeta)_{\omega}\right)$, for any $n$-subset $\omega$ of indices of the columns of $\tilde{M}(\zeta)$ with respect to the $\zeta$-adic valuation $w$. By construction we have

$$
\begin{aligned}
q(\omega)=w\left(\operatorname{det} \tilde{M}(\zeta)_{\omega}\right) & =w\left(\operatorname{det} M\left(\zeta^{N}\right)_{\omega}\right) \\
& =w\left(\left(\operatorname{det} M(\zeta)_{\omega}\right)^{N}\right) \\
& =N w\left(\operatorname{det} M(\zeta)_{\omega}\right) \\
& =N p(\omega) .
\end{aligned}
$$

The lattice $N_{H}=\sum_{i=1}^{m} \mathcal{O}_{H} \zeta^{-u_{i}} v_{i} \otimes 1$ has tropical representation in $L_{q}$ as the projection of the point $u \in \mathbb{Z}^{m}$ with respect to the matroid $q=N p$. By corollary 1 we have $m\left(\nabla_{H}\right)=\min \left\{k \in \mathbb{N} \mid \pi_{N_{H}}\left(u_{k}^{n}\right)=\pi_{N_{H}}\left(u_{k}^{n-1}\right)\right\}$. On the other hand, $\kappa(\nabla)=\frac{1}{N} m\left(\nabla_{H}\right)$ holds. Therefore, we get

$$
\kappa(\nabla)=\min \left\{k \in \frac{1}{N} \mathbb{N} \mid \pi_{\Lambda}\left(u_{k}^{n}\right)=\pi_{\Lambda}\left(u_{k}^{n-1}\right)\right\} .
$$

This formula holds for any extension $H^{\prime}$ of degree divisible by the denominator $s$ of $\kappa(\nabla)$, hence the result also holds in the limit, yielding the claimed result. 
Example (Pflügel-Barkatou). Let $d X / d z=A X$ with

$$
\begin{gathered}
A=\left(\begin{array}{cccc}
-5 z^{-2} & 5 z^{-1} & -2 z^{-1} & -9 z^{-2} \\
5 z^{-3} & 3 z^{-2} & 2 z^{-2} & -4 z^{-2} \\
4 z^{-1} & -5 z^{-1} & -5 z^{-2} & 2 \\
\frac{2-2 z}{z^{3}} & -5 z^{-1} & 3 z^{-2} & -6 z^{-2}
\end{array}\right) . \\
u_{k}^{n}=(0, \ldots,-3 k,-4 k-4 k-4 k-4 k) \\
u_{k}^{n-1}=(0, \ldots,-3 k,-6,-5,-5,-6) . \\
\text { One gets } \pi\left(u_{k}^{n}\right)=\pi\left(u_{k}^{n-1}\right) \Longleftrightarrow k \geqslant \frac{3}{2}, \text { therefore } m(\nabla)=2 \text { but actually } \kappa(\nabla)=\frac{3}{2} .
\end{gathered}
$$

\section{A projection algorithm on a tropical linear space}

The Blue and Red rules from [J-S-Y] recalled in section 3.1 have unfortunately a high computational complexity, since it involves loops over cardinality $\left(\begin{array}{c}m \\ n\end{array}\right)$ sets. In our case, it is especially impractical since for the Gérard-Levelt membrane, we have $m \sim n^{2}$. In this section, we present an efficient algorithm, inspired by Ardila's work on ordinary matroids $\overline{\mathrm{Ar}}$, to compute the projection of a point $x \in \mathbb{R}^{m}$ onto the tropical linear space $L_{p}$ attached to a valuated matroid $p$.

\subsection{Valuated matroids}

Let us recall the setup of valuated matroids, and fix the notations that we will use. For the results listed in this section, we refer to $\mathrm{Mu}-\mathrm{Ta}$, although their definition, following [D-T, comes with the opposite sign. Let $E$ be a finite set, and a map $p: 2^{E} \longrightarrow \mathbb{R}_{\infty}=\mathbb{R} \cup\{\infty\}$. Let $\mathcal{B}=\{B \subset E \mid p(B) \neq \infty\}$. The pair $(E, p)$ is a valuated matroid if $\mathcal{B} \neq \emptyset$ and for $B, B^{\prime} \in \mathcal{B}$ and $u \in B \backslash B^{\prime}$ there exists $v \in B^{\prime} \backslash B$ such that

$$
p(B)+p\left(B^{\prime}\right) \geqslant p(B \cup\{v\} \backslash\{u\})+p\left(B^{\prime} \cup\{u\} \backslash\{v\}\right) .
$$

A subset $B \in \mathcal{B}$ is called a basis of $p$. In particular, $\mathcal{B}$ is the set of bases of an ordinary matroid $P$ on $E$, that we call the matroid underlying $p$. Any vector of the form

$$
X(B, v)=(p(B \cup\{v\} \backslash\{u\})-p(B), u \in E)
$$

for some basis $B$ and $v \in E \backslash B$ is a circuit of $p$. If $X$ is a circuit of $p$, its support

$$
\bar{X}=\left\{e \in E \mid X_{e} \neq \infty\right\}
$$

is a circuit of the matroid $P$. More precisely, it is the fundamental circuit of $B$ and $v$, that is the unique circuit of $P$ included in $B \cup\{v\}$. Similarly, any vector of the form

$$
X^{*}(B, v)=(p(B \cup\{u\} \backslash\{v\})-p(B), u \in E)
$$

for some basis $B$ and $v \in B$ is thus a cocircuit of $p$.

Some important features of circuits and cocircuits of $p$ are in fact encoded in the underlying matroid $P$. For any circuit $C$ of $P$, the set of circuits of $p$ that have $C$ as support is of the form

$$
X+\alpha(1, \ldots, 1) \text { for } \alpha \in \mathbb{R} .
$$

Conversely, for any circuit $X$ of $p, X+\alpha(1, \ldots, 1)$ for $\alpha \in \mathbb{R}$ is a circuit of $p$. The same result applies to cocircuits. Recall the following result. 
Lemma 3. Any circuit (resp. cocircuit) of $P$ containing $v \in E$ can be represented as the fundamental circuit (resp. cocircuit) of a basis $B$ such that $v \notin B$ (resp. $v \in B$ ).

Proof. Let $C$ be a circuit of $P$. By definition, for any $v \in C$, the set $C \backslash\{v\}$ is contained in some basis $B$. Therefore $C \subset B \cup\{v\}$ holds. But there is a unique circuit satisfying this condition. Since the cocircuits are the circuits of the dual matroid, the same result holds.

In what follows, we will speak by abuse of notation of the fundamental (co-)circuit of $B$ and $v$ for a valuated matroid $p$. This is harmless as long as the results that we state are invariant up to the addition of a constant. If we need to specify a representative, we will often use the only one with non-negative coordinates and with minimum coordinate equal to 0 , or with some fixed value at some element of $E$.

For any $x \in \mathbb{R}^{m}$, the map

$$
p_{x}(B)=p(B)-\sum_{b \in B} x_{b}
$$

extended to all $2^{E}$ by $p_{x}(A)=\infty$ for $A \notin \mathcal{B}$ defines another valuated matroid on $E$.

Lemma 4. If $X$ is any circuit of $p$, then $X+x$ is a circuit of $p_{x}$, and if $X^{*}$ is a cocircuit of $p$, then $X^{*}-x$ is a cocircuit of $p_{x}$.

Proof. By the definition of a circuit of $p$, circuits of $p_{x}$ have coordinates

$$
\begin{aligned}
X_{x}(B, v)_{u} & =p_{x}(B \cup\{v\} \backslash\{u\})-p_{x}(B) \text { for some } B \not \supset v \\
& =p(\cup\{v\} \backslash\{u\})-\sum_{b \in B \cup\{v\} \backslash\{u\}} x_{b}-p(B)+\sum_{b \in B} x_{b} \\
& =X(B, v)_{u}-x_{v}+x_{u} .
\end{aligned}
$$

Hence, $X_{x}(B, v)=X(B, v)+x-x_{v}(1, \ldots, 1)$. Similarly, we have

$$
\begin{aligned}
X_{x}^{*}(B, v)_{u} & =p_{x}(B \cup\{u\} \backslash\{v\})-p_{x}(B) \\
& =p(\cup\{u\} \backslash\{v\})-\sum_{b \in B \cup\{u\} \backslash\{v\}} x_{b}-p(B)+\sum_{b \in B} x_{b} \\
& =X^{*}(B, v)_{u}+x_{v}-x_{u} .
\end{aligned}
$$

Hence, $X_{x}^{*}(B, v)=X(B, v)-x+x_{v}(1, \ldots, 1)$. By the projectivity property of circuits and cocircuits, the result is established. Since the sets of bases for $p$ and $p_{x}$ coincide, these are indeed the only (co)circuits of $p_{x}$.

\subsection{An algorithm for the projection on the tight-span}

A valuated matroid $p:\left(\begin{array}{l}E \\ n\end{array}\right) \longrightarrow \mathbb{R}_{\infty}$ of rank $n$ over a finite set $E=[m]$ induces a tropical linear space $L_{p}$ defined by (8). This subspace of $\mathbb{R}_{\infty}^{m}$ corresponds (up to sign) to what Dress and Terhalle call the tight span of a valuated matroid. In this section, we present an efficient algorithmic method to compute the tropical projection from $\mathbb{R}^{m}$ onto $L_{p}$, that generalises results obtained by Ardila for ordinary matroids in $[\mathrm{Ar}]$.

Proposition 2. Let $p$ be a valuated matroid of rank $n$ on $[m]$, and let $u \in E$. The following conditions are equivalent.

i) $u$ belongs to at least one minimal basis of $p$. 
ii) $u$ is never the unique minimum in a circuit of $p$.

iii) $u$ is minimal in some cocircuit of $p$.

Proof. (i) $\Rightarrow$ iiil): Assume that $B$ is a minimal basis containing $u$. Let $C^{*}=X^{*}(B, u)$ be the fundamental cocircuit of $B$ and $u$. By definition, we have

$$
C_{v}^{*}=p(B \cup\{v\} \backslash\{u\})-p(B) \geqslant 0=C_{u}^{*} .
$$

That is, $u$ is minimal in the cocircuit of $B$ and $u$.

iiii) $\Rightarrow$ ii $)$ : suppose that $u$ in the unique minimum for $p$ on a circuit $C$. Assume that $C^{*}$ is a cocircuit of $p$ where $u$ is minimal. By assumption, we have

$$
C_{u}<C_{u^{\prime}} \text { and } C_{u}^{*} \leqslant C_{u^{\prime}}^{*} \text { for } u^{\prime} \neq u .
$$

Accordingly, $C+C^{*}$ has a unique minimum at $u$. By orthogonality of circuits and cocircuits ( $\mathrm{Mu}-\mathrm{Ta}$, th. 3.11, p. 204), the set of indices that minimise $C+C^{*}$ cannot have cardinality one. Therefore, the contradiction is established.

Let us finally prove $[$ ii $\Rightarrow$ i : consider a minimum basis $B$. If $u \notin B$, let $C=$ $X(B, u)$ be the circuit generated by $B$ and $u$. By assumption, the minimum in $C$ is attained at $v \neq u$. The support of $C$ is equal to the fundamental circuit of $B$ and $u$ for the ordinary matroid underlying $p$. Therefore, $B \cup\{u\} \backslash\{v\}$ is a basis of $p$ and

$$
p(B \cup\{u\} \backslash\{v\})-p(B) \leqslant p\left(B \cup\left\{u^{\prime}\right\} \backslash\{v\}\right)-p(B) \text { for } u^{\prime} \in C .
$$

Putting $v=u^{\prime}$ we get $p(B \cup\{u\} \backslash\{v\}) \leqslant p(B)$. Since we assumed that $B$ was minimal, we get (i).

Therefore we get the following characterisation of the (finite part of the) tropical linear space $L_{p}$.

Proposition 3. Let $x \in \mathbb{R}^{m}$, and let $p$ be a valuated matroid of rank $n$ on $[m]$. The following are equivalent.

i) $x \in L_{p}$.

ii) Every element of $E$ belongs at least to one x-minimal basis of $p$.

iii) Every circuit of $p$ contains at least $2 x$-minimal elements.

iv) Every element of $E$ is x-minimal in at least one cocircuit of $p$.

Proof. (i) and [iii) are equivalent by the definition of $L_{p}$ (cf. [J-S-Y]). The remaining assertions are obtained by applying proposition 2 to the valuated matroid $p_{x}$.

Note that the previous characterisation does not apply when $x$ has an infinite coordinate, since $p_{x}$ is then no longer a valuated matroid. However, $x_{u}=\infty$ happens only when $u$ does not belong to any basis.

The computation of $\pi_{L_{p}}(x)$ can be performed independently for every element of the vector $x$. For a given $u \in E$, there is a (unique) normalisation of a circuit $C$ of $p$ containing $u$ such that $C_{u}^{x}=x_{u}$.

Proposition 4. If $u \in E$ violates any one of the three conditions of proposition 国 for the matroid $p_{x}$, then $u$ satisfies them for the modified vector $x^{\prime}=\left(x_{1}, \ldots, x_{u}^{\prime}, \ldots, x_{m}\right)$ with

$$
x_{u}^{\prime}=\max _{u \in C} \min _{e \in C \backslash\{u\}} C_{e}^{x},
$$

where all the circuits are normalised so that $C_{u}^{x}=x_{u}$. Moreover, the conditions of proposition Q are not satisfied at $u$ for $x^{\prime \prime}=\left(x_{1}, \ldots, x_{u}^{\prime}-\varepsilon, \ldots, x_{m}\right)$ with $\varepsilon>0$. 
Proof. By assumption, $u$ is the unique $x$-minimum over some circuit $\widetilde{C}$ containing $u$. The support of such a circuit $C$ can be defined as $\bar{C}=X(B, u)$ the fundamental circuit of $u$ and a basis $B \not \ngtr u$. The $x$-value at $e \in \bar{C}$ of the circuit $C$ is of the form

$$
C_{e}^{x}=p(B \cup\{u\} \backslash\{e\})-p(B)+x_{e}+\alpha \text { for some constant } \alpha \in \mathbb{R},
$$

so we may choose as representative of any circuit $C$ containing $u$ the only one such that $C_{u}^{x}=x_{u}$, namely the one defined by $C_{e}^{x}=p(B \cup\{u\} \backslash\{e\})-p(B)+x_{e}$.

Then by assumption

$$
x_{u}^{\prime} \geqslant \min _{e \in C \backslash\{u\}} \widetilde{C}_{e}^{x}>\widetilde{C}_{u}^{x}=x_{u}
$$

and for any circuit $C \ni u$, we have

$$
C_{e}^{x^{\prime}}=\left\{\begin{array}{l}
C_{e}^{x^{\prime}} \text { if } e \neq u \\
C_{u}^{x^{\prime}}=x_{u}^{\prime} \text { if } e=u
\end{array}\right.
$$

Therefore

$$
C_{u}^{x^{\prime}} \geqslant \min _{e \in C \backslash\{u\}} C_{e}^{x}=\min _{e \in C \backslash\{u\}} C_{e}^{x^{\prime}}
$$

so $u$ cannot be the unique $x^{\prime}$-minimum of any circuit containing $u$. On the other hand, there exists a circuit $C$ containing $u$ such that $\min _{e \in C \backslash\{u\}} C_{e}^{x}=x_{u}^{\prime}$. Putting $x_{u}^{\prime \prime}=x_{u}^{\prime}-\varepsilon$ for any $\varepsilon>0$, then $u$ will be the $x^{\prime \prime}$-unique minimum over the circuit $C$. Thus $x^{\prime}$ is the smallest vector that corrects the value of $x$ at $u$.

Proposition 5. Let $x \in \mathbb{R}^{m}$ and let $B$ be an $x$-minimal basis of $p$. Then $\omega=\pi_{L_{p}}(x)$ can be computed in the following way.

$$
\omega_{i}=\left\{\begin{array}{l}
x_{i} \text { if } i \in B \\
\min _{u \neq i}\left(p(B \cup\{i\} \backslash\{u\})-p(B)+x_{u}\right) \text { otherwise. }
\end{array}\right.
$$

Proof. If $i \in B$ holds, then $i$ is $x$-minimal in the fundamental cocircuit $X^{*}(B, i)$. Therefore all conditions of proposition 2 apply to $i$. Otherwise, let $X(B, i)$ be the fundamental circuit of $B$ and $i$, normalised so that $X(B, i)_{i}=x_{i}$. We have to prove that

$$
\min _{e \neq i}\left(X(B, i)_{e}+x_{e}\right)=\max _{i \in C} \min _{e \in C \backslash\{i\}} C_{e}^{x} .
$$

By construction, $\leqslant$ holds. Actually, it is sufficient to prove that $\min _{e \neq i}\left(X(B, i)_{e}\right) \geqslant$ $\min _{e \in C \backslash\{i\}} C_{e}$ for any circuit $C$ containing $i$.

Say that $\min _{e \neq i} X(B, i)_{e}=X(B, i)_{u}$. For any circuit $C$ containing $i$, there exists $v \in C \backslash\{i\}$ such that $B \cup\{v\} \backslash\{u\}$ is a basis. Since $i \notin B \cup\{v\} \backslash\{u\}$, the circuit $\widetilde{C}=X(B \cup\{v\} \backslash\{u\}, i)$ containing $i$ is well defined, and $\widetilde{C}_{v} \geqslant \min _{e \in \widetilde{C} \backslash\{i\}} \widetilde{C}_{e}$ holds. By definition, we have

$$
\begin{aligned}
\widetilde{C}_{v} & =p((B \cup\{v\} \backslash\{u\}) \cup\{i\} \backslash\{v\})-p(B \cup\{v\} \backslash\{u\}) \\
& =p(B \cup\{i\} \backslash\{u\})-p(B \cup\{v\} \backslash\{u\}) \\
& \leqslant p(B \cup\{i\} \backslash\{u\})-p(B) \text { since } B \text { is minimal } \\
& \leqslant X(B, i)_{u} .
\end{aligned}
$$

Accordingly, we get $X(B, i)_{u} \geqslant \widetilde{C}_{v} \geqslant \min _{e \in \widetilde{C} \backslash\{i\}} \widetilde{C}_{e}$. 
This result implies the following efficient method to compute the tropical projection $\pi_{L_{p}}(x)$ for $x \in \mathbb{R}^{m}$.

1. Compute a minimal basis $B$ of $p_{x}$. This can be performed by the greedy algorithm described in D-W.

2. For $i \in E \backslash B$, compute $C=X(B, i)$. To do this, it suffices to compute the fundamental circuit of $B$ and $i$ for the underlying matroid $P$.

3. Find the minimum element of $C+x$ outside $i$; note that there are at most $n$ non infinite elements to consider.

\section{References}

[Ar] F. Ardila, Subdominant matroid ultrametrics, Annals of Combinatorics, 8, 2004, pp. 379-389.

[B-V] D. G. Babbitt, V. S. Varadarajan, Formal reduction theory of meromorphic differential equations : a group theoretic view, Pacif. J. Math., 109, 1983, pp. 1-80.

[Ba-J-L] W. Balser, W. B. Jurkat, D. A. Lutz, A General Theory of Invariants for Meromorphic Differential Equations, Part I : Formal Invariants, Funkcialaj Ekvacioj, vol. 22, 1979, pp. 197-221.

[C-G-Q] G. Cohen, S. Gaubert, J.P. Quadrat, Duality and Separation Theorems in Idempotent Semimodules, Linear Alg. and Appl., Vol. 379, 2004, pp. 395-422.

[Cor] E. Corel, On Fuchs' relation for linear differential systems, Compositio Math., 140, 2004, pp. 1367-1398.

[De] P. Deligne, Équations différentielles à points singuliers réguliers, Lect. Notes in Math., vol. 163, Springer-Verlag, 1970.

[D-T] A. W. M. Dress, W. Terhalle, The tree of life and other affine buildings, Documenta Mathematica, Extra Volume ICM 1998, Part III, pp. 565-574.

[D-W] A. W. M. Dress, W. Wenzel, Valuated matroid: A new look at the greedy algorithm, Appl. Math. Letters, 3, 1990, pp. 33-35.

[Fu] L. I. Fuchs, Zur Theorie der linearen Differentialgleichungen mit veränderlichen Coeffizienten, J. rein. angew. Math. 66, 1866, pp. 121-160.

[G-K] S. Gaubert, R. Katz, Minimal half-spaces and external representation of tropical polyhedra, J. Alg. Combinatorics, vol. 33, n³, 2011, pp. 325-348.

[G-L] R. Gérard et A. H. M. Levelt, Invariants mesurant l'irrégularité en un point singulier d'un système d'équations différentielles linéaires, Ann. Inst. Fourier, Grenoble, 23 (1), 1973, pp. 157-195.

[Ju] W. Jurkat, Meromorphe Differentialgleichungen, Lect. Notes in Math. 637, Springer Verlag, 1978.

[Ka] N. Katz, Nilpotent connections and the monodromy theorem. Applications of a result of Turrittin, Publ. Math. IHES, 39, 1970, pp. 176-232. 
[H-W] A. Hilali, A. Wazner, Formes super-irréductibles des systèmes différentiels linéaires, Num. Math. 50 (4), 1987, pp. 429-449.

[J-S-Y] M. Joswig, B. Sturmfels, J. Yu, Affine buildings and tropical geometry, Alb. J. Math., 4, pp 187-211.

[K-T] S. Keel, J. Tevelev, Geometry of Chow quotiens of Grassmannians, Duke Math. J. 134, no. 2, 2006, pp. 259-311.

[M] Y. Manin, Moduli fuchsiani, Ann. Sc. Norm. Sup. Pisa, 19 (1) Serie III, 1965, pp. $113-126$.

[Mo] J. Moser, The order of a singularity in Fuchs' theory, Math. Z. 72, 1960, pp. 379-398.

[Mu-Ta] K. Murota, A. Tamura, On circuit valuation of matroids, Adv. Appl. Math., $26, \mathrm{n}^{\circ} 3,2001$, pp. $192-225$.

[W] A. Werner, A tropical view on Bruhat-Tits buildings and their compactifications, Cent. Eur. J. Math. 9, n², 2011, pp. 390-402.

[Y-Y] J. Yu, D. S. Yuster, Representing tropical linear spaces by circuits, Proceedings of FPSAC, 2007. 